\title{
Size Effects on Transport Properties in Topological Anderson Insulators
}

\author{
Wei Li, ${ }^{1}$ Jiadong Zang, ${ }^{1}$ and Yongjin Jiang $^{2}$ \\ ${ }^{1}$ Department of Physics, Fudan University, Shanghai, 200433, People's Republic of China \\ ${ }^{2}$ Center for Statistical and Theoretical Condensed Matter Physics, and Department of Physics, \\ Zhejiang Normal University, Jinhua 321004, People's Republic of China
}

(Dated: September 26, 2018)

\begin{abstract}
We study the size effects on the transport properties in topological Anderson insulators by means of the Landauer-Büttiker formalism combined with the nonequilibrium Green function method. Conductances calculated for serval different widths of the nanoribbons reveal that there is no longer quantized plateaus for narrow nanoribbons. The local spin-resolved current distribution demonstrates that the edge states on the two sides can be coupled, leading to enhancement of backscattering as the width of the nanoribbon decreases, thus destroying the perfect quantization phenomena in the topological Anderson insulator. We also show that the main contribution to the nonquantized conductance also comes from edge states. Experiment proposals on topological Anderson insulator are discussed finally.
\end{abstract}

PACS numbers: 72.15.Rn, 72.25.-b, 03.65.Vf

Recently, the study of the topological insulator (TI) has triggered great research interests $\underline{\underline{1}} \underline{\underline{3}}$. It was first proposed both from topological band theory and topological field theory, and soon experimentally realized in $\mathrm{HgTe} / \mathrm{CdTe}$ quantum wells (QWs) ․․․ $^{\text {. }}$. Although it is insulating in the bulk, conduction is allowed on the boundary due to the presence of edge states. These edge states are protected by the time reversal symmetry. Each edge state is accompanied by its time reversal partner, and the number of pairs is an odd number for topological nontrivial phase, which leads to an odd-integer quantized conductance on each edge. However these stories are restricted to where the TI is semi-infinite. When two edges are getting close to each other, finite size effect ${ }^{6.7}$ plays an important role. The overlap between edge states from opposite edges opens an energy gap so that properties of TI can be subtly modified.

The concept of TI can be generalized into many other insulators, such as topological Mott insulator ${ }^{\underline{8}}$, topological superconductor ${ }^{\underline{9}-11}$. Its generalization in Anderson insulator (AI) is also addressed in disordered system $\underline{\underline{12}} \underline{\underline{15}}$ recently. It is believed that the disorder-induced edge states are topologically protected and play a central role. In this sense such insulator is named as topological AI (TAI). Thus, a question arises naturally that whether this phenomenon will also be emergent in the narrow nanoribbon with finite size. It is interesting to explore the role edge state plays in the finite size system.

In the present paper, we studied size effects on TAI in detail. We choose to study the typical topological band insulator formed by $\mathrm{HgTe} / \mathrm{CdTe} \mathrm{QWs}$, where spin-orbit coupling is encoded. TAI phase is addressed at certain random strength when the Fermi surface is at the bulk conduction band, where a quantized conductance is observed. We show that the conductance is no longer quantized plateaus for narrow nanoribbons. To understand clearly the physics of this picture, we presented the local spin-resolved current distribution in the disordered bar, which demonstrated that the main contribution to the nonquantized conductance comes from edge states. However, due to the truncation of the coherence length between two edges by the finite sample size, disorder can induce the interedge scattering. As a result, the TAI phase will be suppressed. Through detailed size dependence study, we found that by decreasing the width of the nanoribbon, the coupling between edge states will lead to exponential enhancement of the backscattering which destroys the TAI phase eventually.

As a starting point, we consider a $\mathrm{HgTe} / \mathrm{CdTe}$ QWs narrow nanoribbon. The low energy electron states are approximately described by an effective four band Hamiltonian ${ }^{4}$ :

$$
\hat{\mathcal{H}}=\left(\begin{array}{cc}
h(\mathbf{k}) & 0 \\
0 & h^{*}(-\mathbf{k})
\end{array}\right)
$$

where $h(\mathbf{k})=\epsilon(\mathbf{k})+\vec{d}(\mathbf{k}) \cdot \hat{\sigma}, \mathbf{k}=\left(k_{x}, k_{y}\right)$ is the twodimensional wave vector, $\hat{\sigma}=\left(\hat{\sigma}_{x}, \hat{\sigma}_{y}, \hat{\sigma}_{z}\right)$ are Pauli matrices. Up to the lowest order of $\mathbf{k}, \overrightarrow{d(}(\mathbf{k})=$ $\left(A k_{x}, A k_{y}, M-B k^{2}\right)$, and $\epsilon(\mathbf{k})=C-D k^{2}$, where the parameters $\mathrm{A}, \mathrm{B}, \mathrm{C}$ and $\mathrm{D}$ depend on the thickness of $\mathrm{HgTe} / \mathrm{CdTe} \mathrm{QWs} . h^{*}(-\mathbf{k})$ is nothing but the time reversal counterpart of $h(\mathbf{k})$ so that time reversal symmetry is respected. The Hamiltonian is obtained by reducing the eight-band Kane model to the reduced Hilbert space $|E 1,1 / 2>| H 1,,3 / 2>, \mid E 1,-1 / 2>$, and $\mid H 1,-3 / 2>$. Mass $M$ is an important parameter describing the energy gap between conduction and valence band. The Hamiltonian with $M>0$ describes a conventional band insulator, while $M<0$ corresponds to the TI. In the present study, tight-binding lattice model is used, so that the above effective model is compactified by substitutions $k_{i} \rightarrow \frac{1}{a} \sin \left(k_{i} a\right)$, and $k_{i}^{2} \rightarrow \frac{2}{a^{2}}\left(1-\cos \left(k_{i} a\right)\right)$, where $i=x, y$, and $a$ is the lattice constant. The width and length of the nanoribbon under study are $L_{y}$ and $L_{x}$ respectively. Meanwhile, we introduced disorders through random on-site energy with a uniform distribution within $[-W / 2, W / 2]$, with the disorder strength $W$. 


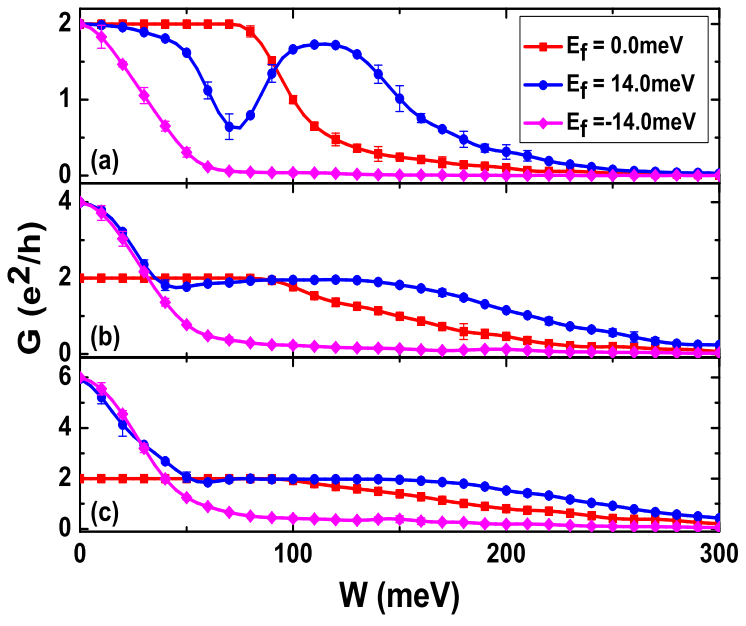

FIG. 1: (Color online) The conductance $G$ (a)-(c) vs disorder strength $W$ for different Fermi energy $E_{f}$. The widths $L_{y}$ of the nanoribbon are (a) $L_{y}=100 \mathrm{~nm}$, (b) $L_{y}=200 \mathrm{~nm}$, and (c) $L_{y}=300 \mathrm{~nm}$.

To calculate the transport properties, we applied the method by nonequilibrium Green function $\frac{14}{}$. A small external bias $V=V_{L}-V_{R}$ is applied longitudinally between the two terminals. The local current between neighboring sites $i$ and $j$ is calculated by the formula $16-19$ :

$$
\left.J_{i \rightarrow j}=\frac{2 e^{2}}{h} \operatorname{Im}\left[\sum_{\alpha \beta} \hat{\mathcal{H}}_{i \alpha, j \beta} G_{i \beta, j \alpha}^{n}\left(E_{f}\right)\right]\left(V_{L}-V_{R}\right)\right]
$$

where $V_{L(R)}$ describes the voltages at the lead-L(R). $G^{n}\left(E_{f}\right)=G^{r} \Gamma_{L} G^{a}$ is electron correlation function with line width function $\Gamma_{L(R)}=i\left[\Sigma_{L(R)}^{r}-\Sigma_{L(R)}^{a}\right]$, and the retarded Green functions $G^{r}\left(E_{f}\right)=\left[G^{a}\left(E_{f}\right)\right]^{\dagger}=1 /\left[E_{f}-\right.$ $\left.H_{c e n}-\Sigma_{L}^{r}-\Sigma_{R}^{r}\right]$, with $H_{c e n}$ the Hamiltonian in the central region. The local spin-resolved current $J_{i \rightarrow j}^{\alpha}$ between neighboring sites $i$ and $j$ with spin index $\alpha$ can also be calculated from Eq. (2) without summing over spin index $\alpha$. The net current $J_{L}$ flowing through the central region is calculated by summing index $i$ for local currents $J_{i \rightarrow i+\hat{x}}$ over any cross-section. After obtaining the current $J_{L}$, the linear conductance is given by $G=\lim _{V \rightarrow 0} d J_{L} / d V$. In addition, the linear conductance can be directly obtained by $G=\operatorname{Tr}\left[\Gamma_{L} G^{r} \Gamma_{R} G^{a}\right]$.

In the following numerical calculations, we used the realistic material parameters of the $\mathrm{HgTe} / \mathrm{CdTe} \mathrm{QWs}^{5}$ : $A=364.5 \mathrm{meV} \cdot \mathrm{nm}, B=-686 \mathrm{meV} \cdot \mathrm{nm}^{2}, C=0 \mathrm{meV}$, $D=-512 \mathrm{meV} \cdot \mathrm{nm}^{2}$, and $M=-10 \mathrm{meV}$. The length of the nanoribbon $L_{x}=1000 \mathrm{~nm}$, and the lattice constant $a=5 \mathrm{~nm}$. Since this model is only valid for small $k$, we set the Fermi energy small around the $\Gamma$ point. In the presence of disorder, the conductance is averaged over up to 400 random configurations except for Fig. 4 where 800 random configurations are used for each data.

We first studied the conductance $G$ versus disorder strength $W$ for different Fermi energy $E_{f}$ and the width

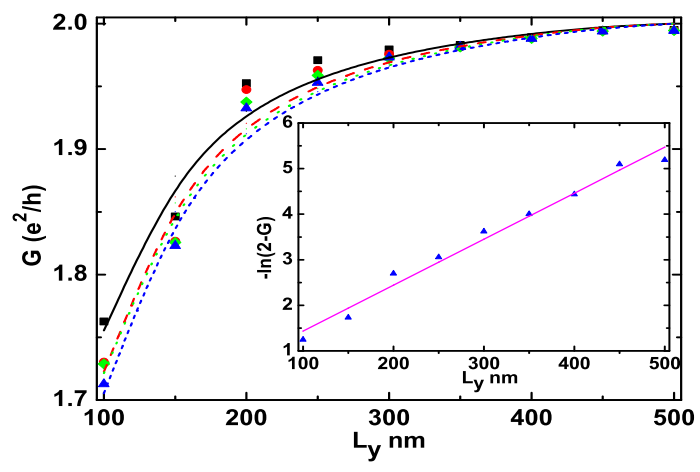

FIG. 2: (Color online) The conductance $G$ vs bar width $L_{y}$ at disorder strength $W=115 \mathrm{meV}$. Different lengths are chosen with $L_{x}=1000 \mathrm{~nm}$ (black solid line), 2500nm (red dash line), 4000nm (green dot line), and 5000nm (blue short dash line), while the symbols $\mathbf{\square}, \bullet$ and $\boldsymbol{\Delta}$ are corresponding to the realistic results of exact diagonalization, respectively. The inset shows the linear fitting of $\ln (2-\mathrm{G})$ by picking a typical length $L_{x}=5000 \mathrm{~nm}$.

$L_{y}$ of the nanoribbon, as shown in Fig 1 As long as the Fermi surface $E_{f}=0.0 \mathrm{meV}$ (see Fig (1) lies inside the bulk gap, the conductance maintains a quantized value $2 e^{2} / h$ for different widths in the clean limit. The conductance remains this quantized value in a broad range of the disorder. When the disorder further increases, electronic states become localized, and the conductance decreases to zero rapidly. Such observation agrees well with the previous result that the quantum spin Hall effect $\underline{20}-\underline{23}$ is robust against weak disorder and independent of the width of the nanoribbon. It's well known that a finite size of the Hall bar opens a gap on the edge due to overlap between edge states 6 . Thus the edge states are no longer Dirac particles. Our result shows that although the edge states gain mass in nanoribbon, the topology is still preserved. Backscattering is still forbidden due to time reversal symmetry according to a brilliant argument in Ref. 1.

When the Fermi surface lies in the valence band of the bulk such as $E_{f}=-14 \mathrm{meV}$ shown in Fig. 1] system is metallic in the absence of disorder. Once the disorder is turned on, electrons become localized, and the conductance dives to zero directly (see Fig. 11). This result exhibits the conventional Anderson localization phenomenon. On the other hand, when the Fermi energy $E_{f}$ is raised up into the bulk conduction band, say $E_{f}=14 \mathrm{meV}$, and the width is sufficiently large [see Fig. 1(b) and (c)], conductance $G$ decreases gradually when the strength of the disorder increases. Comparing to the Fig. 1(b) and (c), it is apparent that the "dip" in conductance (which occurs at $W \sim 60 \mathrm{meV}$ ) becomes less and less pronounced as the nanoribbon width $L_{y}$ increases. Because the increasing of the nanoribbon width $L_{y}$, the number of conducting channels in the bulk is increased. The more conducting channels there are, the larger the conductance will be. In addition, the relation 

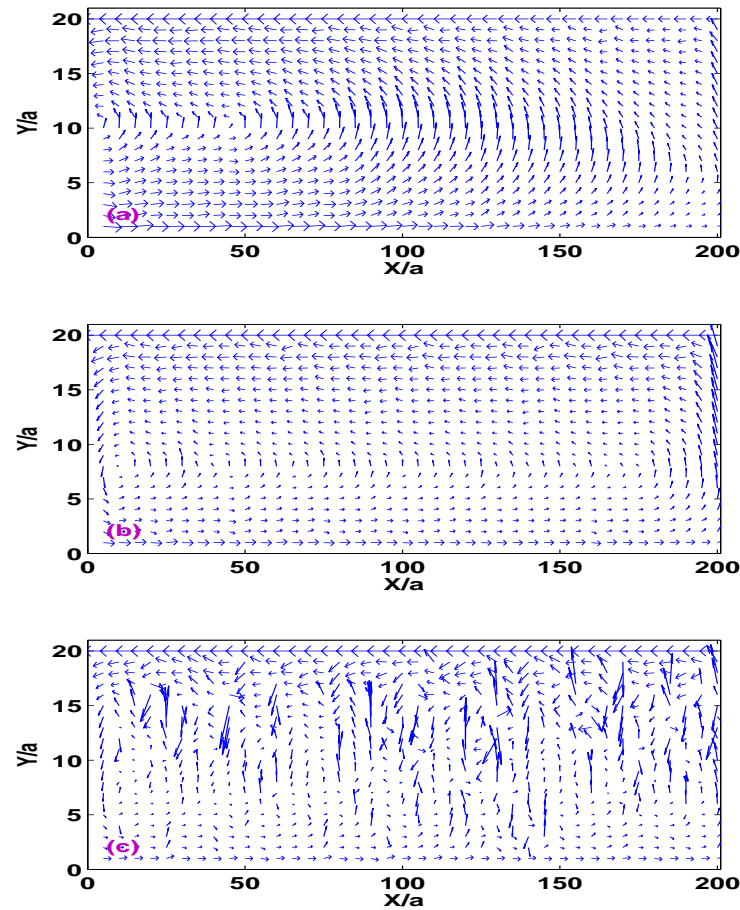

FIG. 3: (Color online) The distribution of local spin-resolved current with disorder strength (a) $W=75.0 \mathrm{meV}$, (b) $W=$ $90.0 \mathrm{meV}$, and (c) $W=115 \mathrm{meV}$. The Fermi energy is $E_{f}=$ $14.0 \mathrm{meV}$, and the bar size is $L_{x}=200 a$, and $L_{y}=20 a$. The vector length is proportional to the square root of the current value.

between conductance $G$ and the nanoribbon width $L_{y}$ obeys: $G L_{x}=\sigma_{c} L_{y}$, where $\sigma_{c}$ is the conductivity, which is width independent. Beyond a certain onset of $W$, the conductance turns back and increases to an approximate quantized value $\left(2 e^{2} / h\right)$. $G$ maintains this value for a certain range of $W$ before eventually decreases. Concerning the spin degeneracy, each spin component contributes one conductance quanta only. This odd-integer valued conductance plateau indicates that the the system is in a topologically distinct phase on this stage. More importantly, different from the pure TI, the quantized value is induced by disorder. This topological nontrivial phase is exactly the TAI phase. However, one should note that when the width is as small as $L_{y}=100 \mathrm{~nm}$, as shown in Fig. 1(a), although the plateau is still present, it is no longer integer quantized, but irrationally valued in unit of $e^{2} / h$. At the same time, the conductance plateau evolves into a hump structure.

To further understand the finite size effect on TAI, the relation between hump peak value and bar size is investigated in detail, as shown in Fig. 2. With the increase of width, the plateau conductance $G$ increases as well, and finally saturates to $2 e^{2} / h$. After numerous calculation, we found that the quantity $(2-G)$ behaves as an exponential function of the width $L_{y}$, which is quite un-

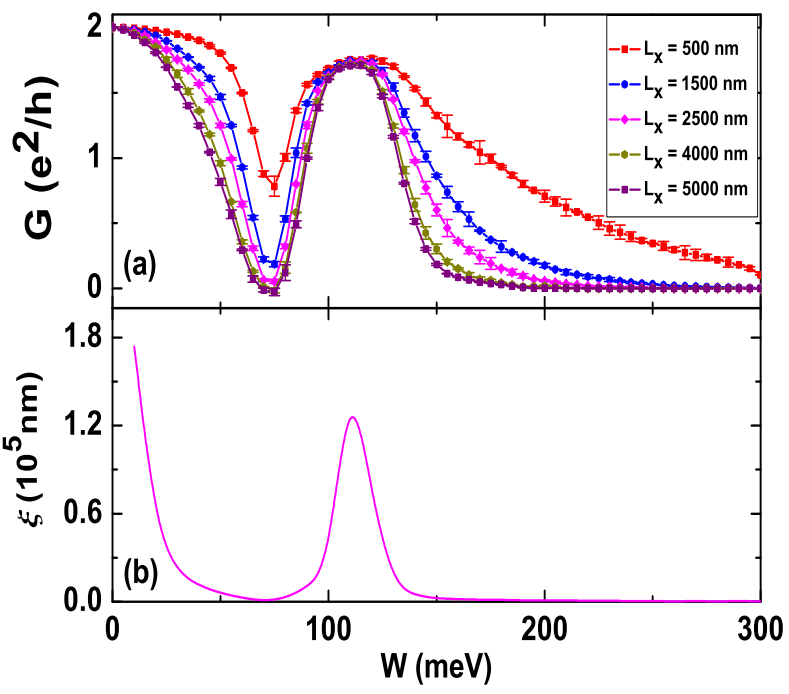

FIG. 4: (Color online) (a) the conductance $G$ vs disorder strength $W$ for different lengths of the nanoribbon. (b) the localization length $\xi$ vs disorder strength $W$. The Fermi energy $E_{f}=14.0 \mathrm{meV}$ and the width of the nanoribbon is $L_{y}=100 \mathrm{~nm}$.

conventional. In the case of normal metal, conductance is proportional to the width due to the increase of channels. This exponential behavior of conductance suggests the conductance hump is contributed also by the edge channel of the Hall bar, as the interaction between states localized on opposite edges decays exponentially with respect to the width. Due to previous studies by Groth et $a l^{13}$, finite disorder would reverse the sign of mass term of the BHZ model ${ }^{4}$, leading to a robust edge state. The above result adds new insights to this understanding in narrow nanoribbon structure.

In order to get a better insight into the microscopic origin of the conductance variations as shown in Fig. 廿(a), the local spin-resolved current distributions are plotted in Fig. 3. Due to the time reversal symmetry, we only consider the spin-up component, the influence of spindown component can be directly obtained by time reversal symmetry. Here, the local spin polarized current on site $i$ is defined as $J_{i}^{\uparrow}=J_{i \rightarrow i+\hat{x}}^{\uparrow}+J_{i \rightarrow i+\hat{y}}^{\uparrow}$. When $W=75.0 \mathrm{meV}$, the whole sample behaves similarly as a conventional metal with large backscattering. The vortex-like circulation pattern can be easily seen, which reflects that the scattering direction are determined by the chirality of spin orbital interaction. As to the transport property, this is an AI as the conductance decreases very rapidly as an exponential form with respect to the bar length $L_{x}$ [see Fig.4(b)]. As the disorder strength increases gradually, current on one edge is greatly suppressed, while enhanced on the other edge. Meanwhile, the bulk of the sample becomes more and more insulating. All these signals indicates a formation of stable edge states. At last, when the disorder is raised 
up to $W=115.0 \mathrm{meV}$ and conductance reaches peak value, bulk states are extremely disordered and insulating. Edge states on the upper edge survives and appears to be very robust against disorder. However, due to the finiteness of the nanoribbon width, small backscattering is still present by hopping from the upper edge to the lower one. The reduction of conductance is apparently proportional to this hopping probability which is an exponential function of the width.

In Fig. 4(a), the conductance $G$ as a function of disorder strength $W$ with different length $L_{x}$ of the nanoribbon are plotted. The dip feature is clearer for a large $L_{x}$ because of the increasing probability of the backscattering between the two edges. It is interesting to point out that the main shape of the conductance hump at around the disorder strength $W=115 \mathrm{meV}$ changes little with the bar length, and the system in that region exhibits the nonquantized version of TAI behavior. The corresponding localization length $\xi=-\lim _{L_{x} \rightarrow \infty} L_{x} / \ln (G)$ as a function of disorder strength $W$ is shown in Fig. 4(b). We notice that the localization length increases dramatically near the conductance hump region, which is the finite size version of the picture that reasonable disorder scattering can drive an insulating system into a TAI phase so that the system shows quantized conductance $\frac{12,13}{2}$. On the other hand, $\xi$ almost vanishes near the dip region. From the theoretical viewpoint, the sharp difference of longitudinal localization length in different disorder regions plays the central central role for all experimental observable effects, both for bulk and finite size version of TAI.

In summary, the size effects on the transport properties in TAI are studied in this paper. We found the conductance plateau deviates from its quantized value exponentially as the nanoribbon width decreases. Such behavior is originated from the mutual interaction between edge states, as is clearly evidenced by local spin-resolved current distributions in the disordered bar. Furthermore, we found the conductance is generally decaying exponentially as the system length increases. However, in the TAI region, the longitudinal localization length is extremely long, which is found up to hundreds of microns. This is quite a good feature that makes the TAI phase with a narrow nanoribbon experimentally distinguishable.

It is reported 24 that topological insulator can be realized in optical lattices, where the disorder can be introduced by an optical laser speckle potential25,26. By tuning the laser intensity, it's quite promising to observe the TAI and the finite size effect. However in contrast to the uniform random disorder studied in this work, the disorder in optical laser speckle is correlated. The effect of this difference is unknown up to now. Therefore, it should be an interesting problem for future studies.

We gratefully acknowledge the financial support from the National Natural Science Foundation of China under grant No.11004174. WL and JZ thank the support by Fudan Research Program on Postgraduates. WL thanks Qing-feng Sun, and Jian Li for helpful discussions.
1 X.-L. Qi, S.-C. Zhang, Physics Today, 63, 33 (2010).

2 M. Z. Hasan, C. L. Kane, Rev. Mod. Phys. 82, 3045 (2010).

3 X.-L. Qi, S.-C. Zhang, arXiv: 1008.2026.

4 B. A. Bernevig, T. L. Hughes, and S. C. Zhang, Science 314, 1757 (2006).

5 M. Konig, S. Wiedmann, C. Brune, A. Roth, H. Buhmann, L. W. Molenkamp, X.-L. Qi, and S.-C. Zhang, Science 318, 766 (2007).

${ }^{6}$ B. Zhou, H.-Z. Lu, R.-L. Chu, S.-Q. Shen, and Q. Niu, Phys. Rev. Lett. 101, 246807 (2008).

7 J. Linder, T. Yokoyama, and A. Sudbø, Phys. Rev. B 80, 205401 (2009).

8 S. Raghu, X.-L. Qi, C. Honerkamp, S.-C. Zhang, Phys. Rev. Lett. 100, 156401 (2008).

9 L. Fu, C. L. Kane, Phys. Rev. Lett. 100, 096407 (2008).

10 L. Fu, C. L. Kane, Phys. Rev. B 79, 161408(R) (2009).

11 L. Fu, C. L. Kane, Phys. Rev. Lett. 102, 216403 (2009).

12 J. Li, R.-L. Chu, J. K. Jain, and S.-Q. Shen, Phys. Rev. Lett. 102, 136806 (2009).

13 C. W. Groth, M. Wimmer, A. R. Akhmerov, J. Tworzydlo, and C. W. J. Beenakker, Phys. Rev. Lett. 103, 196805 (2009).

14 H. Jiang, L. Wang, Q.-F. Sun, X. C. Xie, Phys. Rev. B 80, 165316 (2009).
15 H.-M. Guo, G. Rosenberg, G. Refael, and M. Franz, Phys. Rev. Lett. 105, 216601 (2010).

16 Y. Hatsugai, Phys. Rev. B 48, 11851 (1993).

17 A.-P. Jauho, N. S. Wingreen, and Y. Meir, Phys. Rev. B 50, 5528 (1994).

18 S. Nakanishi and M. Tsukada, Phys. Rev. Lett. 87, 126801 (2001)

19 Y. Zhang, J.-P. Hu, B. A. Bernevig, X. R. Wang, X. C. Xie, and W. M. Liu, Phys. Rev. B 78, 155413 (2008).

20 C. L. Kane and E. J. Mele, Phys. Rev. Lett. 95, 146802 (2005).

21 C. L. Kane and E. J. Mele, Phys. Rev. Lett. 95, 226801 (2005).

22 L. Fu, C. L. Kane, and E. J. Mele, Phys. Rev. Lett. 98, 106803 (2007).

23 L. Sheng, D. N. Sheng, C. S. Ting, and F. D. M. Haldane, Phys. Rev. Lett. 95, 136602 (2005).

${ }^{24}$ B. Béri and N. R. Cooper, arXiv:1105.1252

25 J. E. Lye, L. Fallani, M. Modugno, D. S. Wiersma, C. Fort, and M. Inguscio, Phys. Rev. Lett. 95, 070401 (2005).

26 J. Billy, V. Josse, Z. Zuo, A. Bernard, B. Hambrecht, P. Lugan, D. Clément, L. Sanchez-Palencia, P. Bouyer and A. Aspect, Nature 453, 891 (2008). 